


\title{
DISEÑO Y CONSTRUCCIÓN DE UN SISTEMA ÓPTICO PARA ANÁLISIS ESPECTRAL UTILIZANDO LA TÉCNICA LIF (FLUORESENCIA INDUCIDA POR LUZ)
}

Mario S. López, Luis A. Manzano, Eduardo Ávalos, Rolando Sáenz.

Escuela Politécnica Nacional

Recibido: 10 - noviembre - 2015, aprobado 03 - diciembre - 2015

\section{Resumen}

En este artículo se presentan los resultados preliminares de medidas de fluorescencia con base en un espectrómetro construido para uso de la técnica LiF. Este proyecto consiste en el diseño y construcción de un espectrómetro portable con una fuente luminosa ultravioleta, con el fin de producir fluorescencia sobre ciertas sustancias líquidas transparentes, como por ejemplo las relacionadas con el petróleo. El equipo de medición consta de una fuente luminosa que está compuesta por dispositivos electrónicos y ópticos que generan y enfocan luz ultravioleta, un sistema de energía para que pueda ser portátil, y un sistema de adquisición de datos que permitirá el análisis de fluorescencia en las muestras.

Palabras clave: fluorescencia, espectrómetro, ultravioleta, petróleo, análisis.

\begin{abstract}
This article presents the preliminary results of fluorescence measurements based on a spectrometer, built for using of $\mathrm{LiF}$ technique. This project consists in the designing and construction of a portable spectrometer with ultraviolet light source, in order to produce fluorescence upon certain transparent liquid substances, such as the related with the oil. The measuring equipment consists of a light source that is composed of electronic and optical devices which generate and focus ultraviolet light, a power system so it can be portable, and a data acquisition system, that will allow fluorescence analysis samples.
\end{abstract}

Keywords: fluorescence, spectrometer, ultraviolet, oil, analysis. 


\section{Introducción}

Basado en la aplicación que tiene la luz, muy estudiada en muchos campos por sus aplicaciones diversas que van desde entretenimiento hasta la curación de ciertas enfermedades y análisis espectral de materiales, se desarrolló este proyecto tecnológico

En la aplicación diseñada en este proyecto se pretende crear un espectrómetro con una fuente luminosa ultravioleta a base de un diodo emisor de luz, capaz de excitar o perturbar ciertos materiales para generar fluorescencia por trasmisión. Para ello se ha empleado un conjunto de elementos eléctricos, electrónicos, mecánicos, sistemas de energía y lentes ópticos, cuyo objetivo es el de perturbar un material predeterminado con luz enfocada emitida por el diodo emisor de luz ultravioleta. La información obtenida es procesada mediante un sistema informático.

\section{Desarrollo De Contenidos}

La fuente luminosa requiere de varios elementos para lograr fluorescencia, en primer lugar el elemento activo que genera la luz ultravioleta: un diodo LED UV, el cual es un elemento electrónico. El sistema eléctrico/electrónico del sistema consiste en una fuente de alimentación (batería), protecciones eléctricas como reguladores de tensión, PTC, y el microcontrolador el que realizará las tareas de censado de batería, modo de funcionamiento del LED, etc.

Otro punto importante de la fuente es el sistema óptico, el mismo que está conformado por un lente divergente, su función es la de enfocar la luz generada por el LED (arreglo de 9 emisores de luz) en un solo punto, éste se proyecta sobre la muestra a analizar, es móvil en un rango de $1 \mathrm{~cm}$.

Para realizar el análisis espectral se debe emplear un fluorosensor, componente fundamental del instrumento de medida denominado espectrómetro. La luz enfocada sobre la muestra se modifica por la absorción de la sustancia fluorescente, emitiendo radiación de mayor longitud de onda y de menor energía [1]. La Figura 1 indica las etapas del sistema espectroscópico.

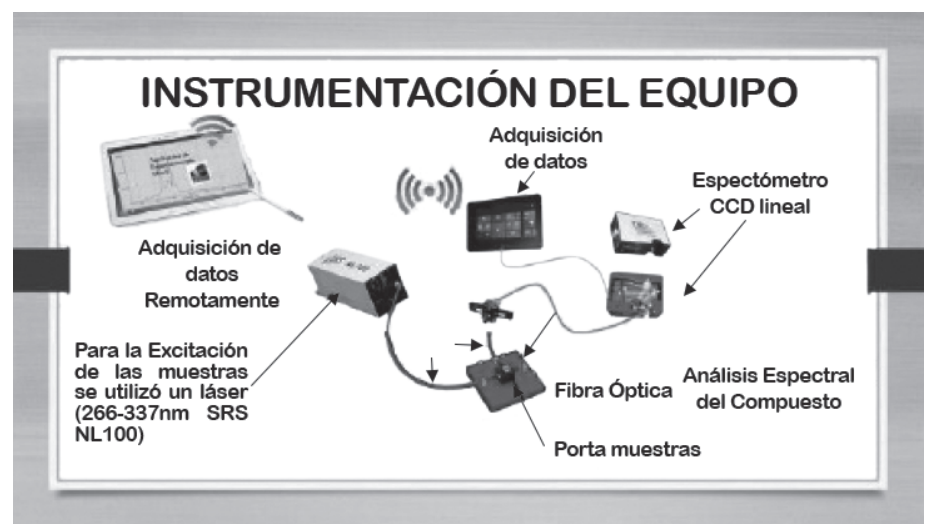

Figura 1. Etapas del sistema espectroscópico. 


\section{Instrumentación Óptica}

\section{A. Led Uv}

El diodo emisor de luz de la empresa ROHITNER modelo LED UVLUX 320-5 es el utilizado en este trabajo para la generación de luz ultravioleta, la característica fundamental de este LED es que la longitud de onda de la luz emitida es de alrededor de $330 \mathrm{~nm}$ y su potencia óptica (potencia radiante) es de 3 a $5 \mathrm{~mW}$. La Figura 2 señala su potencia radiante en función del ángulo de radiación de este elemento. El ángulo de radiación es importante para el diseño del lente que enfoca la luz emitida por el LED.

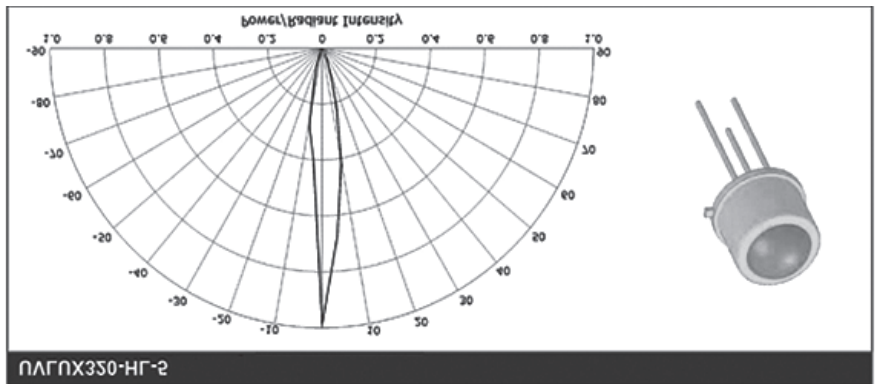

Figura 2. Potencia radiante con ángulo de radiación del LED UVLUX 320-5.

\section{B. Diseño de lente de enfoque}

Tomando en cuenta los parámetros ópticos del diodo LED UV, se procede al cálculo del lente necesario para poder enfocar su luz en un solo punto. Mediante conceptos básicos de radiometría y óptica geométrica, se puede realizar una aproximación matemática de los requerimientos del lente. La Figura 3, indica un esquema general para el diseño del lente en base a la distancia focal, apertura y ángulo de radiación.

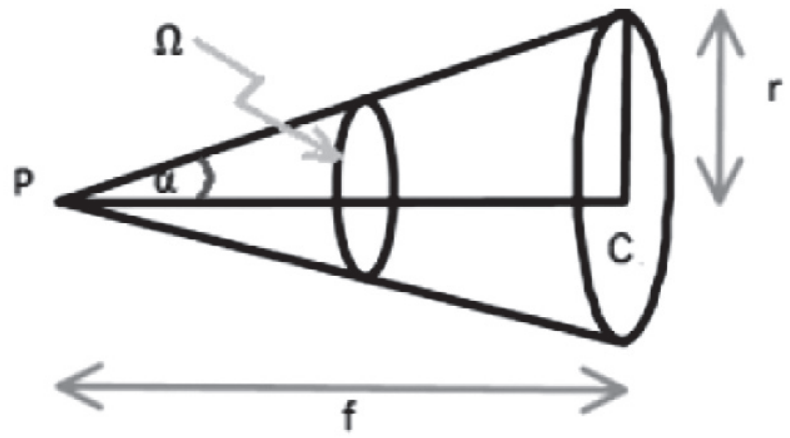

Figura 3. Ángulo sólido subtendido por una lente desde su foco.

Para realizar el cálculo del diámetro mínimo del lente, debemos asumir la distancia $f$, la longitud desde el LED hasta el lente; se tomará una distancia variable en el rango de 2 a 3 centímetros para que el operario pueda ajustar el enfoque de acuerdo a sus necesidades. Se calcula la distancia r con el triángulo rectángulo for- 
mado por $\mathrm{P}, \mathrm{r}$ y $\mathrm{C}$, de acuerdo al ángulo de radiación dado por el lente hemisférico propio del LED, cuyo valor es de 20 grados. El ángulo alfa $\alpha$ tendrá un valor de 10 grados por ser su mitad.

Una vez encontrado el valor de apertura del lente se pueden calcular los radios de curvatura del lente divergente con un índice de refracción de 1,89 que corresponde a un vidrio Flint pesado [2].

El lente se moverá mediante un riel mecánico adaptado a un motor DC, el mismo que generará el movimiento deseado mediante dos pulsadores, un pulsador hará que el motor gire en sentido horario y el otro en sentido anti horario, con esto se puede controlar el movimiento del lente. Para limitar la distancia de movimiento se colocan sensores finales de carrera, los mismos que al activarse desconectan al motor DC de la alimentación para impedir el movimiento del mismo.

\section{Sistema eléctrico y electrónico}

\section{A. Batería}

Es indispensable tener una fuente de energía para poder activar todos los elementos eléctricos y electrónicos del sistema.

Las baterías son elementos activos que dan voltaje DC a su salida. Existen muchos tipos de batería por lo cual se debe hacer un análisis de cuáles utilizar.

Para este proyecto se ha seleccionado una batería de polímero de litio (LiPo) recargable de 3 celdas, que es una variación de la batería de ion litio (Li-ion). Como características, tienen gran capacidad de corriente, presentan una tasa alta de descarga, es decir, podemos sacar mucha corriente en poco tiempo, físicamente son más pequeñas que otro tipo de baterías y por ende pesan menos, lo cual es indispensable para la portabilidad del sistema.

1) Censado y monitoreo de batería: Generalmente los sistemas autónomos (alimentados por baterías recargables) tienden a mostrar al usuario el nivel de batería disponible, con el fin de conocer el tiempo de funcionamiento restante del sistema. Para el caso de esta aplicación se utiliza el circuito mostrado en la Figura 4, con el fin de conocer en tiempo real el porcentaje de carga de batería mediante el uso de un puerto de conversión análogo/digital de un microprocesador. Mediante software se realiza la transformación de los valores numéricos obtenidos de la conversión $\mathrm{A} / \mathrm{D}$ para poder visualizar en un LCD el porcentaje real de carga de batería.

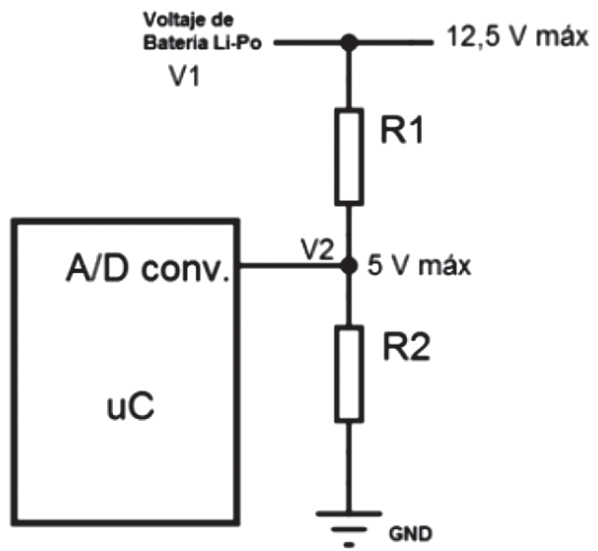

Figura 4. Divisor de voltaje para limitación de voltaje y corriente al pin del conversor A/D del microcontrolador. 
2) Autodesconexión de batería: Para mantener la vida útil de una batería tipo LiPo, no se debe permitir que el voltaje descienda de cierto nivel mínimo recomendado por el fabricante, ya que en caso contrario podría sufrir daños irreparables. Es por eso que se debe diseñar un circuito que desconecte automáticamente la batería al llegar a un voltaje mínimo establecido, la Figura 5 muestra a este circuito. Los voltajes de operación que se han establecido son de $12,5 \mathrm{~V}$ para un porcentaje de carga de $100 \%$ y $10 \mathrm{~V}$ para un porcentaje de $0 \%$. Es decir, cuando la batería presente un voltaje menor a $10 \mathrm{~V}$, el circuito debe desconectar la batería del sistema.

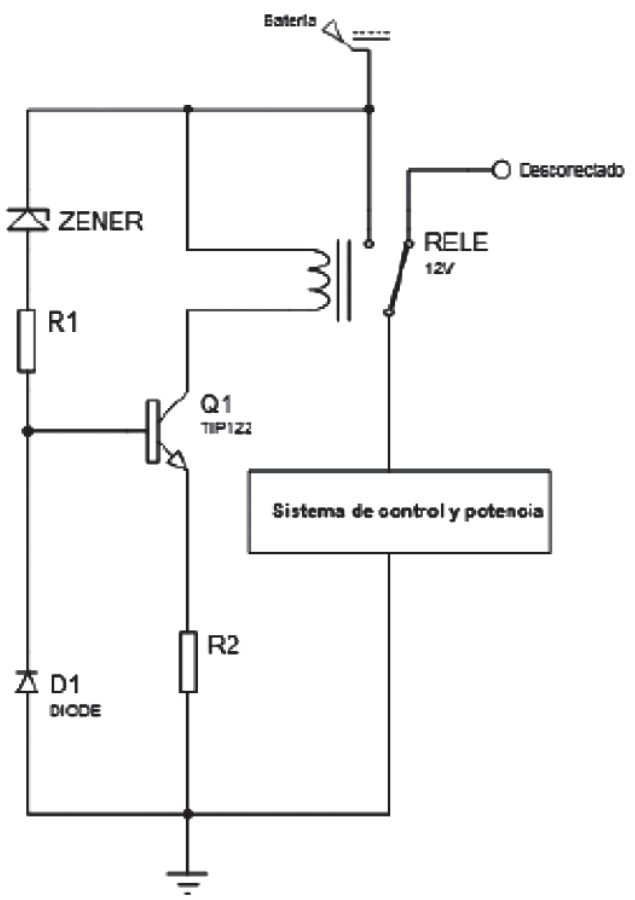

Figura 5. Circuito de auto-desconexión de batería.

\section{B. Microcontrolador ATmega8}

Es un microcontrolador CMOS de 8 bits de bajo consumo, basado en la arquitectura RISC mejorada. Sus instrucciones se ejecutan en un ciclo de máquina, el ATmega8 consigue transferencia de información de alrededor de 1 mips por $\mathrm{MHz}$ admitido por el sistema, permitiendo al diseñador del sistema optimizar el consumo de energía versus la velocidad de procesamiento [3].

Se ha utilizado este dispositivo debido a sus numerosas prestaciones y se adapta de mejor manera a las necesidades del proyecto, pues posee un convertidor ADC, por su alta fiabilidad y su disponibilidad en el medio.

\section{Protecciones}

Las protecciones se emplean para garantizar que el comportamiento dinámico de la energía, como picos de corriente, voltaje, sobrecorriente, etc., no afecte en el comportamiento del sistema. Las protecciones empleadas en el proyecto son reguladores de voltaje y fusible reutilizable PTC: 
Mediante un regulador de voltaje se asegura que la alimentación del microcontrolador sea de $5 \mathrm{~V}$ constantes lo que garantiza su correcto funcionamiento. También se coloca otro regulador de $9 \mathrm{~V}$ para asegurar al circuito de conmutación de los LED UV. El regulador de voltaje impide también la aparición de picos de voltaje que atentan contra la integridad del dispositivo encargado del control. El PTC protege de cortos-circuitos que puedan afectar al sistema.

\section{Driver LED}

Los requerimientos de alimentación que presenta el LED UV empleado demandan que se lo haga mediante una fuente de corriente constante, puesto que las fuentes de voltaje facultan la aparición de picos de corriente. Para ello se emplean reguladores de voltaje LM317 en una configuración especial, con la que se logra obtener una fuente de corriente constante. En la Figura 6 se presenta esta configuración.

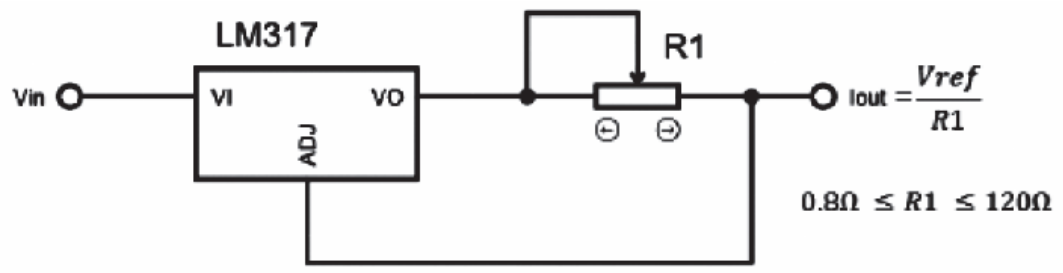

Figura 6. LM317 como fuente de corriente constante [4].

Debido a que el diodo LED funciona tanto de manera continua como intermitente y el microcontrolador no puede satisfacer la demanda de corriente del LED UV para su funcionamiento, se debe crear un circuito que pueda entregar la corriente necesaria, que trabaje de manera continua o intermitente. Para ello se elabora el circuito mostrado en la Figura 7, el cual toma la señal del microcontrolador y la amplifica mediante un transistor bipolar de juntura para disparar un mosfet.

El sistema de potencia funciona con

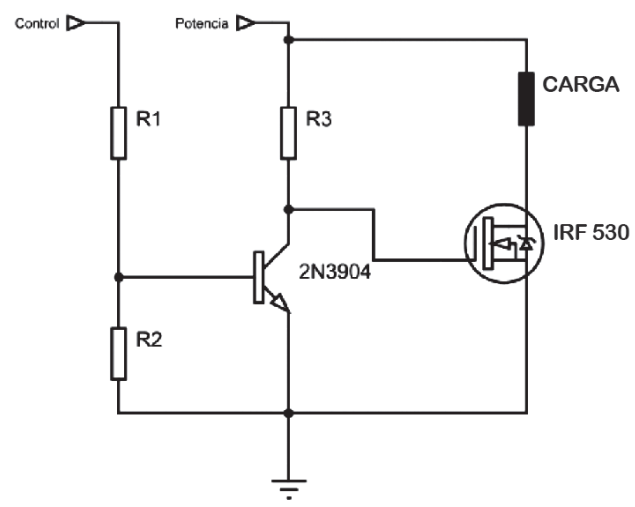

Figura 7. Circuito de conmutación par LED-UV. un voltaje de $9 \mathrm{~V}$, el mismo que proviene de un regulador LM7809 para evitar picos de sobrevoltaje que puede dañar a los LED UV.

\section{E. Display y elementos de control}

1) Censado y monitoreo de batería: Para conocer el nivel de carga que tiene la batería se implementa un LCD de 2 filas por 16 caracteres alfanuméricos, el mismo 
que indica el modo de funcionamiento del sistema (continuo e intermitente) y además el porcentaje de batería. La Figura 8 muestra el LCD que se utiliza en la aplicación, que tiene como característica su bajo consumo energético y su simplicidad para mostrar la información que el usuario necesita conocer.

2) Pulsadores, potenciómetros e interruptor de 3 vias: Para seleccionar el modo de funcionamiento del LED UV se ha empleado pulsadores normalmente abiertos, los cuales físicamente son botones que al presionarlos permiten el paso de corriente eléctrica. En nuestro, caso para
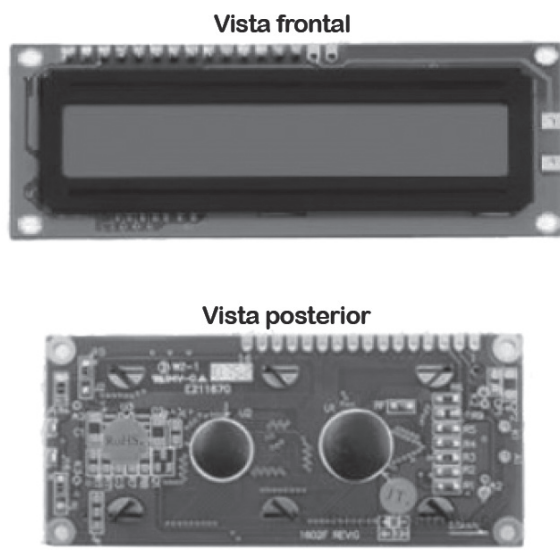

Figura 8. Vista frontal y posterior del LCD de 2 filas y 16 caracteres. conocer el estado de un pin del microcontrolador y asociarlo con el modo de funcionamiento.

El potenciómetro, es una resistencia eléctrica variable, para ello está adaptado a un sistema mecánico que permite seleccionar el valor de resistencia. Mediante el principio de funcionamiento de este elemento podemos controlar el voltaje que ingresa al pin de conversión análogo digital del microcontrolador. Este dispositivo permite controlar la frecuencia del disparo del LED UV.

El interruptor de 3 vías permite encender, apagar y ajustar el sistema para que la batería pueda cargarse. Es un elemento importante en cualquier circuito electrónico ya que limita el funcionamiento continuo del aparato, es decir, si no se lo utiliza se lo puede apagar y si se requiere carga de batería, se la puede realizar sin ningún problema. Esto sirve para alargar la vida útil de todos los elementos del sistema.

La Figura 9 indica un esquema general de cómo se conectan todos los elementos previamente mencionados para su funcionamiento.

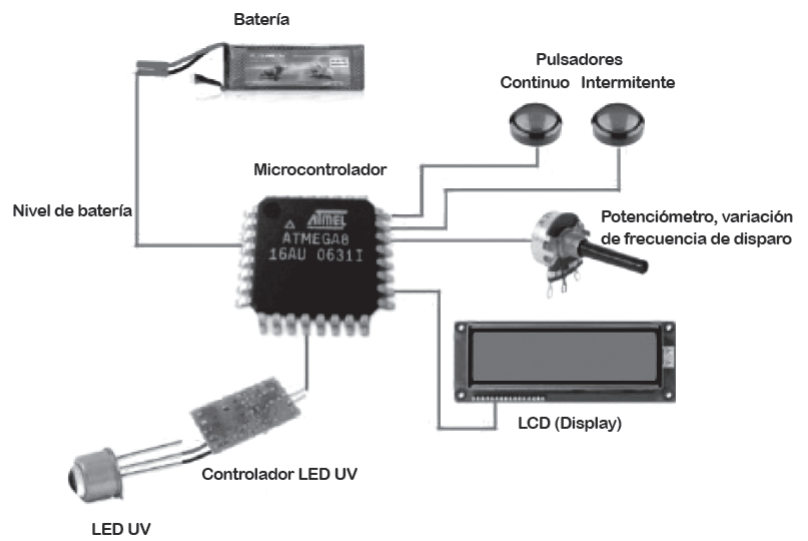

Figura 9. Vista frontal y posterior del LCD de 2 filas y 16 caracteres. 


\section{F. Diseño de la placa SMD}

La tecnología de montaje superficial es un método de construcción actual de dispositivos electrónicos que tiene como ventaja reducir sustancialmente el tamaño de los circuitos electrónicos comunes, ya que emplean dispositivos de montaje superficial que son más eficientes y de baja temperatura.

El circuito de control fue elaborado con esta tecnología para reducir peso, tamaño e incrementar la portabilidad de todo el equipo. Para ello se debió diseñar el sistema en un programa computacional denominado Altium Designer. Este software proporciona las facilidades necesarias para toda clase de diseño electrónico.

La creación del circuito en este programa se consiguió siguiendo varios pasos, entre los más importantes están el diseño del circuito con los elementos que se requieren, eligiéndolos desde una base de datos del programa, en el área de trabajo esquemático se colocan todos los elementos y se conectan de acuerdo al requerimiento del circuito. Una vez obtenido el diagrama esquemático, el mismo que se visualiza en la Figura 10, se genera el documento PCB (Printed Circuit Board) en el cual debemos ajustar el tamaño de pistas, ubicación de elementos, etc. El PCB del circuito de control se puede observar en la Figura 11.

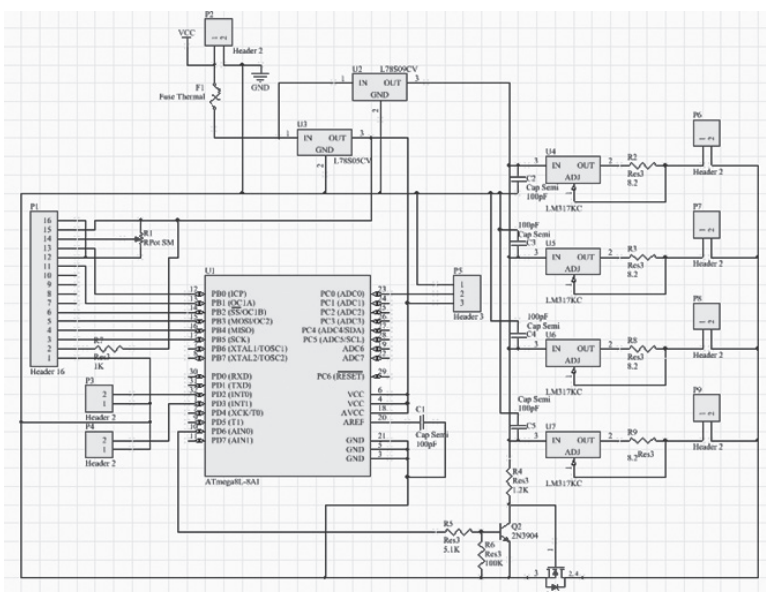

Figura 10. Circuito electrónico esquemático de la placa de control implementado con el programa computacional Altium Designer

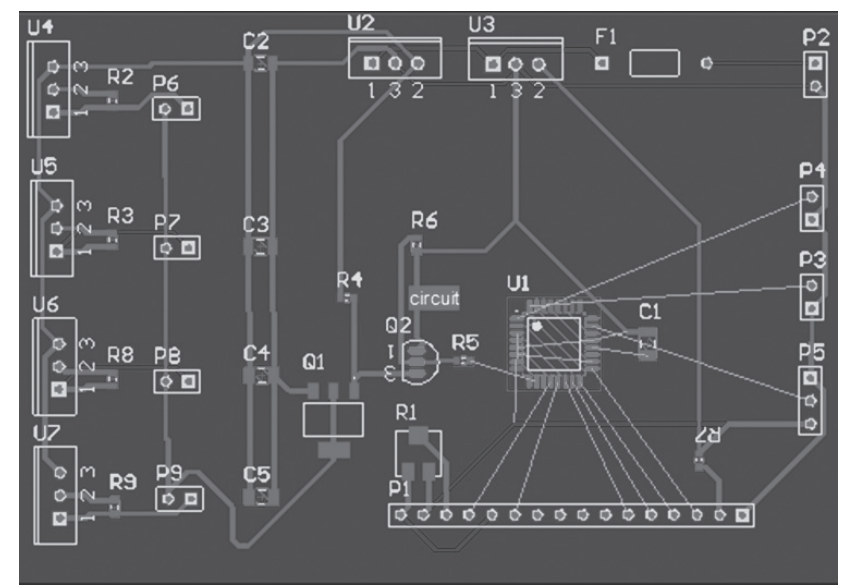

Figura 11. Representación PCB del circuito. 


\section{Software}

\section{A. Código de programación}

El código de programación está realizado en el paquete computacional Bascom AVR. Esta plataforma permite editar códigos en lenguaje Basic particularmente para microcontroladores Atmel AVR.

Entre las ventajas ofrecidas por el programa está la versatilidad que presenta para la configuración de los diversos recursos que tienen los micro controladores AVR, tales como el conversor $\mathrm{A} / \mathrm{D}$, las interrupciones externas, salida de datos a mostradores como LCD, etc. El principal inconveniente que presenta este compilador es que demanda mucha memoria del micro controlador respecto a otros lenguajes de bajo nivel como el ensamblador. Sin embargo para la aplicación no se requiere del manejo de grandes cantidades de datos para su procesamiento, y por ello emplearemos este lenguaje debido a su alta eficacia y facilidad de programación.

Como se dijo anteriormente, el proyecto utiliza la configuración del LCD, la activación y configuración del conversor análogo digital para el control en la frecuencia de disparo y para la medición de la batería, la habilitación de las interrupciones externas para poder realizar el cambio en modo de operación.

\section{B. Display LCD, interfaz de usuario}

El display sirve para mostrar al usuario los modos de operación y el nivel de la batería. Es importante que el usuario conozca esta información para determinar el tiempo de funcionamiento aproximado del dispositivo, como también del modo de operación de los diodos emisores de luz para la aplicación específica.

A continuación se detallan las pantallas de bienvenida y posteriormente la pantalla que se mostrará en funcionamiento normal del LCD.

1) Primera pantalla: En la primera pantalla se muestra una carátula con el título “PROYECTO DE TITULACIÓN”, Figura 12.

\section{PROYECTO DE \\ TITULACION E}

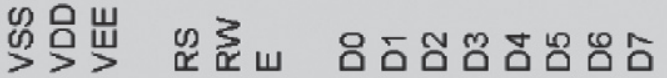

Figura 12. Pantalla de bienvenida 1.

2) Segunda pantalla: En la segunda pantalla se muestra el nombre de los autores, Figura 13. 


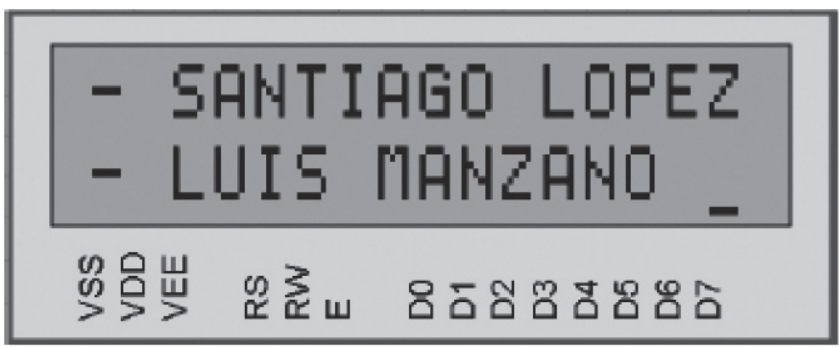

Figura 13. Pantalla de bienvenida 2.

3) Pantalla 3, 4 y 5: En las próximas tres pantallas se muestra un breve manual en el cual se indican las dos opciones de funcionamiento para que el usuario seleccione en base a pulsadores el modo de operación adecuado a sus necesidades, la Figura 14 señala estas pantallas. Cada pantalla tarda 2 segundos en mostrarse, las pantallas de bienvenida solamente se reproducirán al energizar el sistema. Mientras no se seleccione un modo de funcionamiento, las pantallas 3, 4 y 5 indicadas en la Figura 14 se repetirán hasta apoyar sobre uno de los dos pulsadores de selección del modo requerido.

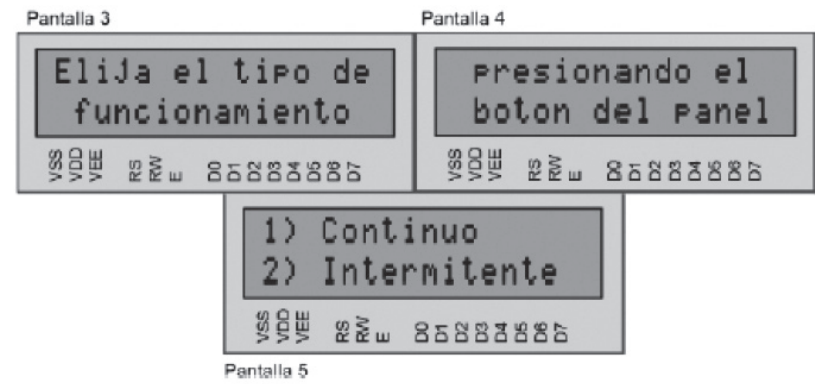

Figura 14. Indicaciones de cómo seleccionar el modo de operación del LED UV.

4) Pantalla de funcionamiento normal: Una vez seleccionado el modo de funcionamiento de la fuente luminosa, la pantalla o display indicará el nivel de carga de batería que alimenta al sistema y el modo de funcionamiento continuo o intermitente que ha sido seleccionado con los pulsadores. La Figura 15 muestra las pantallas en modo de funcionamiento normal.

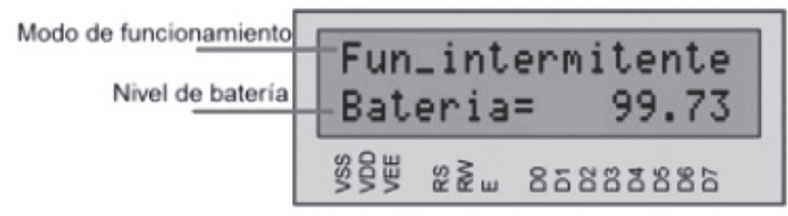

Modo de funcionamiento intermitente

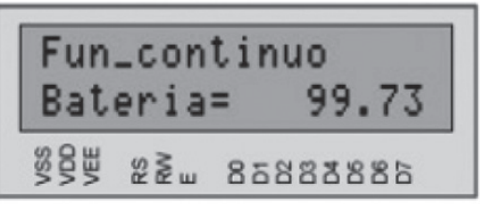

Modo de funcionamiento continuo

Figura 15. Pantallas en modo de funcionamiento normal 


\section{Variación de frecuencia de diodos LED UV}

En el código se deberán programar los tiempos para variar en el rango deseado de frecuencia, para ello el potenciómetro permite variar entre un voltaje de 0 a $5 \mathrm{~V}$ que dará lugar a tener en el convertidor análogo/digital valores numéricos de $0 \mathrm{a}$ 1023 o, de manera digital, desde 0000000000 a 1111111111 . Estos valores pueden ser almacenados en registros temporales que permitirán hacer operaciones matemáticas para tener el valor deseado de frecuencia.

\section{Nivel de batería}

De la misma manera, para la parte de monitoreo de la batería se utiliza el convertidor $\mathrm{A} / \mathrm{D}$ pero sin potenciómetro, ya que la variable digital es el voltaje que suministra la batería, acondicionado de 0 a $5 \mathrm{~V}$. Como en el caso anterior, se tiene en él microcontroladores de 0 a 1023, pero como la batería nunca llega a descargarse a valores menores de $9 \mathrm{~V}$, se limitará por software el rango de carga y descarga de la batería. El voltaje máximo de descarga para nuestra aplicación es de $10 \mathrm{~V}$ y el voltaje máximo de carga de $12.5 \mathrm{~V}$. Esto significa que en el puerto A/D del microcontrolador introduciremos un voltaje máximo de $5 \mathrm{~V}$ para $100 \%$ de carga de la batería y $4 \mathrm{~V}$ para $0 \%$ de carga de la batería.

\section{E. Aplicación de la fuente de luz con un espectrómetro}

La fuente de luz de este proyecto se diseñó con el fin de alimentar a un sistema más grande y complejo. A continuación se dará una breve explicación del funcionamiento de todo el sistema para conocer la aplicación y alcance que puede llegar a tener.

El proceso consta de cinco etapas: la primera es la perturbación de la muestra, seguida por el ingreso de la información desde la muestra al espectrómetro a consecuencia de la perturbación. En la etapa tercera se realiza el procesamiento de la información en el interior del espectrómetro. La cuarta etapa establece el enlace de comunicación y, finalmente, se interpreta la señal proveniente del espectrómetro mediante el software para que sea susceptible de análisis para el operador.

Para la perturbación se emplea la fuente luminosa creada en base a los UV leds. El haz de luz ultravioleta obtenido incide sobre la muestra. El medio que se usa para transportar la luz desde la fuente a la muestra es el medio circundante ya que la toma de información se la hace por transmisión. En este punto, considerando que la entrada del espectrómetro disponible es un terminal con conector SMA, el tubo de ensayo que contiene las muestras para analizar deberá estar lo más próximo al mismo para permitir que la mayor cantidad de información posible sea interpretada por el espectrómetro.

Cuando la luz incide sobre la muestra, en ésta se genera fluorescencia. La radiación causada por la fluorescencia es captada por el espectrómetro ubicado diametralmente opuesto a la fuente luminosa.

Una vez ingresada al espectrómetro, la radiación es sometida al proceso mediante el cual se traduce como una señal eléctrica. 
Cuando se perturba la muestra en forma intermitente, los resultados erróneos se pueden eliminar, ya que la fluorescencia generada termina por estabilizarse y al espectrómetro ingresa únicamente esta información.

La variación de frecuencia no sería necesaria si toda la materia se comportara de la misma manera, pero la complejidad de los enlaces moleculares conlleva mayores demandas energéticas para alcanzar fluorescencia. Por este motivo los disparos pueden aumentar su frecuencia para aumentar la potencia de perturbación; y si no es posible con esto la producción de fluorescencia, el modo de operación continua es el último recurso, a riesgo de conseguir distorsión en la información final.

Toda información generada en el espectrómetro es enviada a un dispositivo wireless que la empaqueta y permite el envío de la información inalámbricamente. Cuando se establece la conexión con dispositivo operado con Windows, como una tablet o una laptop, interviene el software para procesar la información.

\section{F. Simulación}

Una vez realizados los diseños de los circuitos y el detalle de su funcionamiento, se realiza la simulación mediante el software ISIS de Proteus. La Figura 16 indica el modo intermitente de funcionamiento del LED UV.
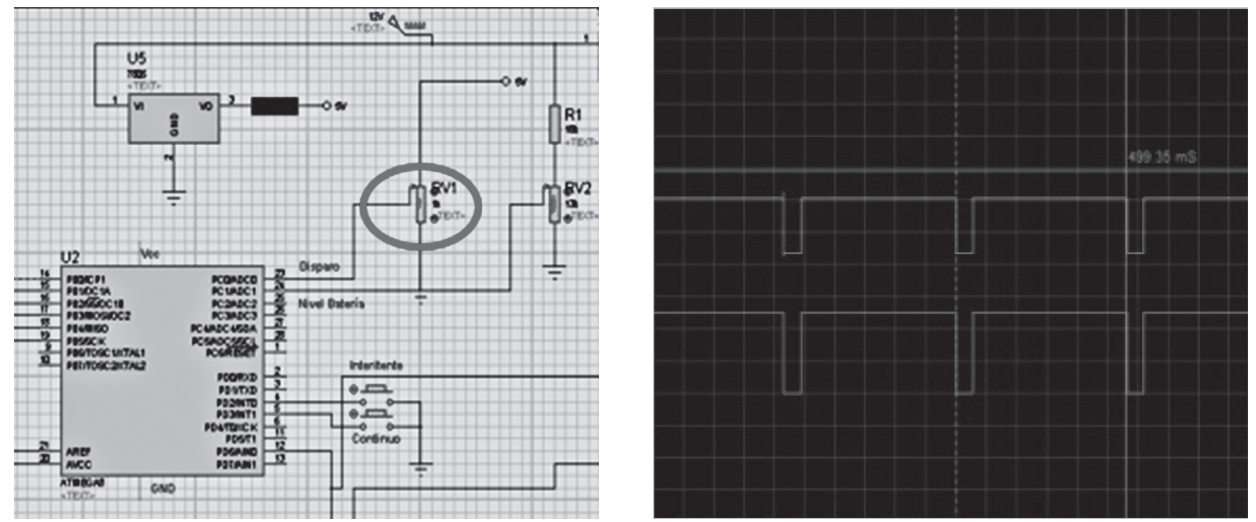

Figura 16. Modo intermitente de LED, variación de frecuencia mediante potenciómetro.

La Figura 17 muestra el cambio del modo de funcionamiento intermitente a un funcionamiento continuo.
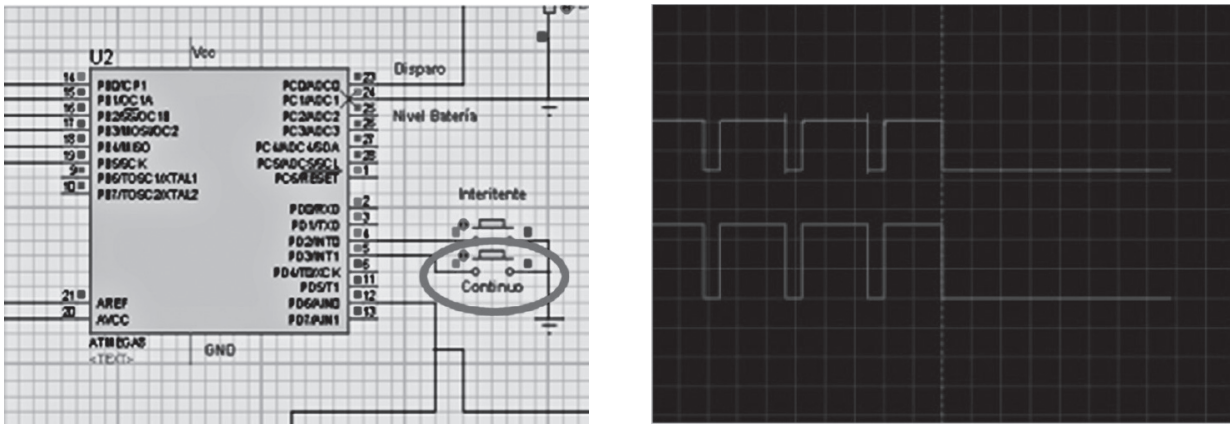

Figura 17. Funcionamiento continuo de LED UV 
Cuando el porcentaje de batería disminuye del $0 \%$ el circuito de conmutación actúa automáticamente evitando que la batería siga descargándose. La Figura 18 indica el mensaje que muestra el LCD antes de apagarse el sistema de manera automática.
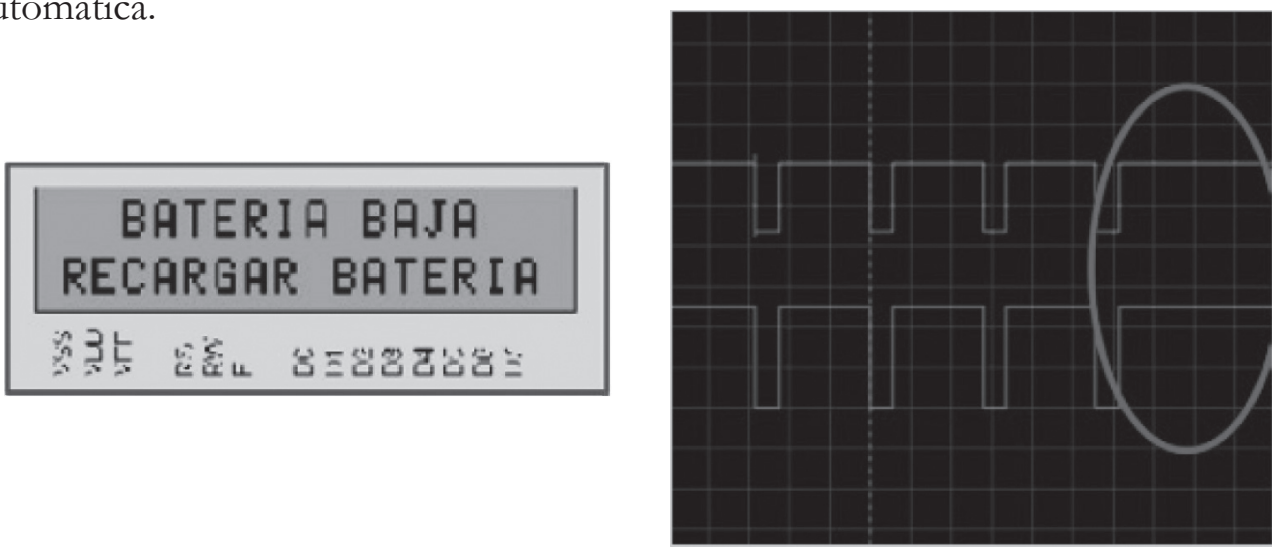

Figura 18. Mensaje de batería baja, porcentaje de carga $0 \%$.

La Figura 19 muestra como el circuito de autodesconexión de la batería actúa cuando se tiene un porcentaje de carga menor al $0 \%$. de batería en carga menor al $0 \%$.

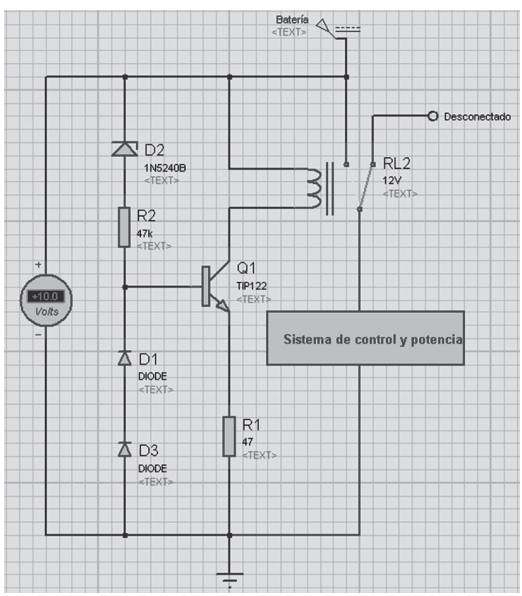

\section{Pruebas y resultados}

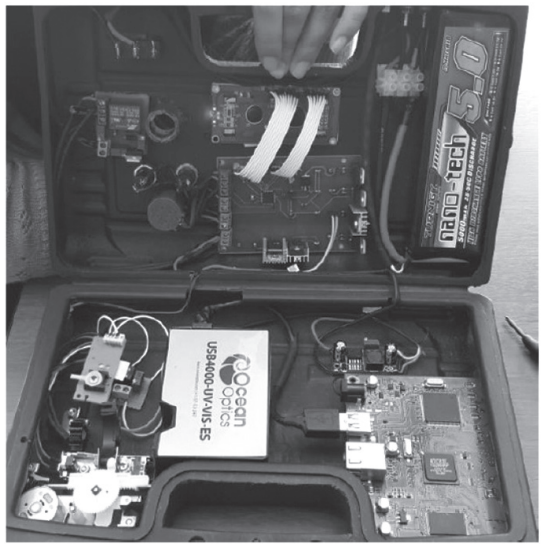

Para comprobar el correcto funcionamiento del sistema se realizaron diferentes pruebas.

\section{A. Ensamble del sistema}

La Figura 20 indica el sistema final ensamblado, en el cual se observan claramente los elementos mecánicos, eléctricos y electrónicos.

Figura 20. Ensamble del sistema completo. 


\section{B. Tiempo de autonomía del sistema}

Realizando pruebas de descarga de batería al amperaje que el sistema demanda se pudo determinar que el tiempo de autonomía es de alrededor de 9 horas de funcionamiento continuo, lo cual es vital para un equipo portátil.

\section{Prueba de fuente pulsada con frecuencia ajustable}

Para el funcionamiento del modo intermitente se requiere que el LED se encienda y se apague periódicamente, de tal manera que el usuario pueda modificar este parámetro. En el osciloscopio se verifica la frecuencia mínima y máxima. Las Figuras 21 y 22 señalan la medición de las frecuencias obtenidas.

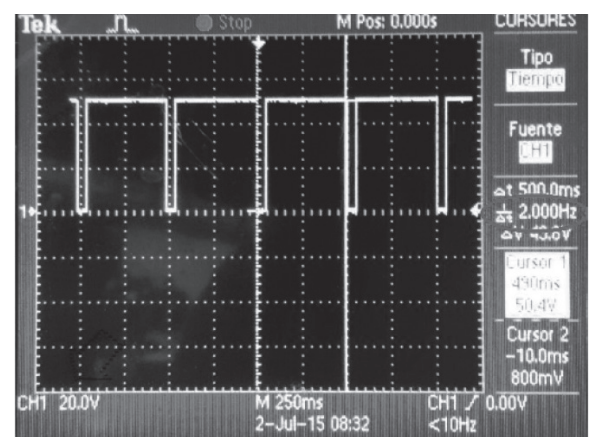

Figura 21. Prueba de frecuencia de switcheo de LED UV a valor.

\section{Prueba de espectro adquirido del LED UV}

Para conocer el espectro a la salida del sistema óptico acoplado, se coloca un espectrómetro de marca Ocean Optics modelo USB4000, el mismo que está ubicado junto a la fuente luminosa, de manera que la luz de la fuente se enfoque sobre el lente del espectrómetro.

La Figura 23 indica el espectro obtenido del LED UV.

\section{E. Pruebas con materiales fluorescentes}

Una vez obtenido el espectro de la fuente luminosa, se realizan pruebas para determinar el espectro fluorescente de otras sustancias ante la luz UV.

Para la toma de espectros se debe proceder con la medición como se señala en la Figura 24.

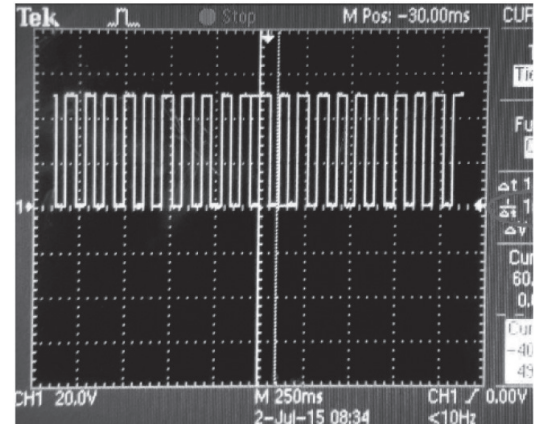

Figura 22. Prueba de frecuencia de switcheo de LED UV a valor máximo del potenciómetro.

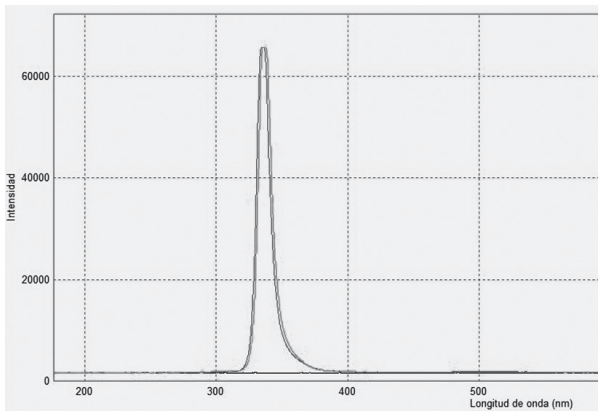

Figura 23. Espectro de LED UV.

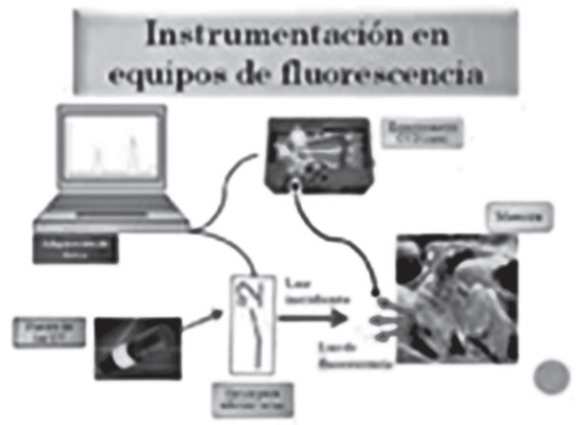

Figura 24. Esquema de adquisición de espectro de muestras líquidas por el método de transmisión.. 
Para validar la fuente se realiza pruebas con distintas sustancias, en donde se obtienen distintos espectros tal como se muestran en las Figuras 25, 26 y 27.

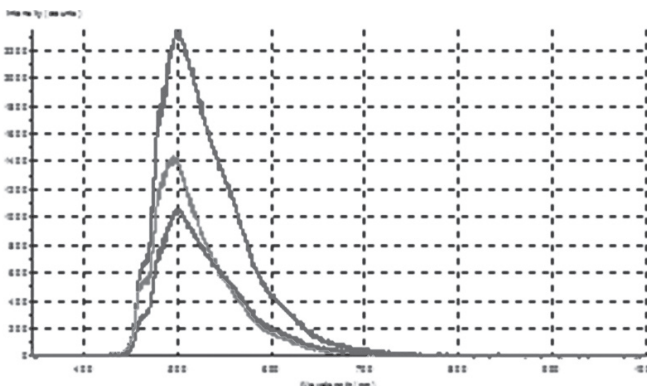

Figura 25. Distribución de luz fluorescente de las gasolinas distribuidas en el Ecuador. Los espectros son representados con diferentes tipos de color: a) Diesel (verde), b) Súper (rosado), c) Extra (rojo)

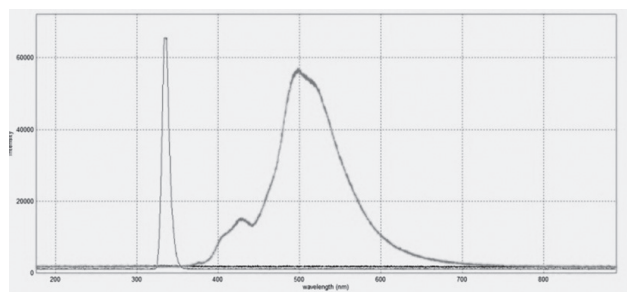

Figura 26. Espectro de combustible Diesel.

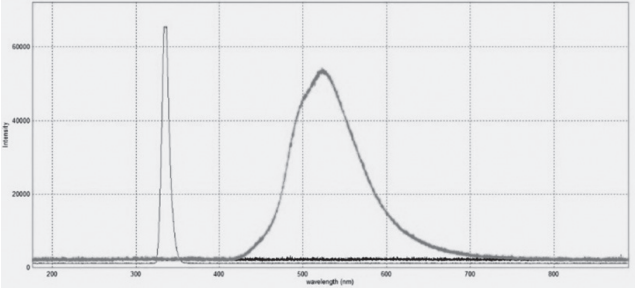

Figura 25. Espectro de agua residual producto del refinamiento del petróleo.

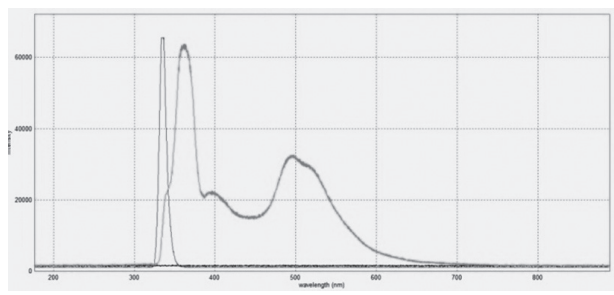

Figura 27. Espectro de gasolina Extra.

\section{Conclusiones}

Se ha logrado implementar un espectrómetro con una fuente luminosa portátil y enfocable de corta distancia UV a base de un arreglo de diodos emisores de luz ultravioletas, que puede excitar materiales fluorescentes para su análisis espectral mediante un espectrómetro.

El LED utilizado es altamente sensible a la estática y a la calidad de energía tanto en voltaje como en corriente que se le suministre. Por tanto, se alimenta por una fuente de corriente constante, lo que permite tener niveles estables en los parámetros antes mencionados. Además, se emplearon reguladores de voltaje y protección de corriente (PTC) para proteger a todo el sistema.

La interfaz para el usuario, tanto electrónica como mecánica, se ha orientado a ser lo más simple posible para facilitar al usuario su manejo. De esta manera se posibilita que personal sin conocimiento profundo de electrónica o espectroscopia (haciendo referencia el área de análisis e interpretación de los resultados que se puedan obtener con el proyecto) lo pueda utilizar, incrementando su versatilidad. 


\section{Bibliografía}

[1] Technical Note: An introduction to fluorescence measurements, Turner Designs Co., 1990, p. 1.

[2] Emilio Gómez González, Radiometría y fotometría, 1 Ed, Departamento de Física Aplicada III, Universidad de Sevilla, 2006, pp. 4-19.

[3] Atmel ATmega8/8L, Atmel Co, San Jose, USA, 2013.

[4] LM317/NCV317, ON Semiconductor Company, Arizona, USA, april 2007. 